\title{
AGUSTÍN ZAVALA*
}

La misión de la filosofía y el problema de la carne en Visible e invisible de M. Merleau-Ponty

\section{A. PROYECTO}

M erleau-Ponty nos ofrece una manera de filosofar que abre V dimensiones desconocidas hasta entonces en la filosofia occidental. Nos dice: "Lo que llamamos carne no tiene nombre en ninguna filosofia". 1 Pero antes de presentarnos una filosofia que "quiere llevar a la expresión las cosas mismas desde el fondo de su silencio",2 es necesario examinar aquellas posibilidades que se le ofrecían y lo que Merleau-Ponty piensa de ellas. Es necesario ver dentro de qué marco quiere filosofar Merleau-Ponty.

\section{Problemas}

Podemos decir que hay una primera zona en que vemos los problemas a que una tarea así se enfrenta. Estos problemas son tres, como tres modalidades de llegar a las cosas mismas en el pensar occidental:

a) La ciencia. La ciencia busca lo objetivo: "lo objetivo es lo verdaderon3 y se mueve en los parámetros de la medición, el orden de hecho y sus variables que definen una posición a los entes, y "las relaciones posibles entre objetos dentro de ese orden".. Pero esta aproximación a las cosas no es lo vivido: "la ciencia comienza por excluir todos los predicados que les tocan a las cosas por

"Centro de Estudios de las Tradiciones, de El Colegio de Michoacán.

${ }^{1}$ Maurice Merleau-Ponty, Le visible et l'invisible, NRF, Editions Gallimard, 1964, p. 193. A menos de que se indique otra cosa, todas las referencias son a esta obra, por eso solamente se anotarán las páginas. Las traducciones son mías.

${ }^{2}$ Ibid, p. 18.

${ }^{3}$ Ibid, p.31.

${ }^{4}$ Cfr. p. 31. 
nuestro encuentro con ellas". ${ }^{5}$ Esta exclusión es provisional y luego se reintroduce lo excluido como "caso particular de las relaciones y de los objetos que para ella definen el mundo". ${ }^{6}$ Sin embargo, por una parte, "la ciencia está enraizada en la pre-ciencia" ${ }^{n 7}$, por otra, aún maneja una trasposición de categorías de una filosofia anticuada: "el físico continúa considerándose Espíritu Absoluto frente al objeto puro", 8 aún tiene una "ontología del Kosmotheoros y de su correlativo el Gran objeto". ${ }^{9}$ La ciencia ya ahora se ve llevada, por una parte, a una manera de ver en la que 'objetivo' y 'subjetivo' son reconocidos como dos órdenes constituidos a la carrera en el interior de una experiencia total cuyo contexto es necesario reconstruir con toda claridad ${ }^{10} \mathrm{y}$, por otra, la ciencia en su modalidad actual "es extraña a la cuestión del sentido de ser". ${ }^{11}$ Es, por lo tanto, incompleta y no ofrece la posibilidad de realizar la filosofia a la manera de Merleau-Ponty. Frente a la ciencia "el mundo percibido está más acá o más allá de la antinomia" de sujeto y objeto ${ }^{12}$ y por esto la tarea es "primeramente saber qué sea un 'mundo,",13 y "reexaminar la definición del cuerpo como objeto puro". 14

b) La filosofia reflexiva. "Por la conversión reflexiva, percibir e imaginar ya no son sino dos maneras de pensar" ${ }^{n}{ }^{15}$ Sin embargo, esto no niega que la filosofia reflexiva sea interrogación. Por el contrario, "la interrogación filosófica y la explicitación resultante, la filosofia reflexiva las practica en un estilo que no es el único posible". ${ }^{16}$ "La reflexión... aclara todo menos su propio papel" $^{117}$ de allí que la filosofía reflexiva no sea una buena alternativa frente a la misión de la filosofia. Ni aún la duda cartesiana, que se mueve dentro de la reflexión, soluciona los problemas, ya que "o bien la duda no es sino un despedazamiento y oscuridad, y entonces no me enseña nada -0 si me enseña algo, es que es deliberada, militante, sistemática... es el orden de los actos, dentro del cual ya quedo para siempre encerrado". ${ }^{18}$ Como otra posibilidad tenemos la aprehensión de esencias. Pero la esencia es una invariante dentro de un orden y "nada nos garantiza que toda experiencia pueda ser expresada en variantes esenciales". ${ }^{19}$ De allí surgen varias dificultades: "por la reflexión el yo perdido en sus percepciones se reencuentra al reencotrarlas como pensamien-

$\begin{array}{lll}{ }^{5} \mathrm{p} .31 . & { }^{10} \mathrm{p} .38 . & { }^{15} \mathrm{p} .49 . \\ { }^{6} \mathrm{Ibid} . & { }^{11} \mathrm{p} .33 . & 16 \mathrm{p} .54 . \\ { }^{7 \mathrm{p} .33 .} & 12 \mathrm{p} .41 . & 17 \mathrm{p} .55 . \\ { }^{8} \mathrm{p} .32 . & 13 \mathrm{p} .47 . & 18 \mathrm{p} .59 . \\ { }^{9} \mathrm{p} .35 . & { }^{14} \mathrm{Ibid.} & 19 \mathrm{p} .70 .\end{array}$


tos" 20 y "la filosofía reflexiva reemplaza el 'mundo' por el 'entrepensar", 21 es decir, "la filosofía reflexiva transforma de un solo golpe el mundo efectivo en campo trascendental". ${ }^{22} \mathrm{Y}$ así tenemos que "la filosofia reflexiva... es triplemente infiel a aquello que se propone esclarecer: al mundo visible, al que lo ve, y a sus relaciones con otros 'visionarios'". ${ }^{23}$ Surge entonces la pregunta: "¿es necesario entrar en la reflexión?"24 y la respuesta es: "lo que nos proponemos es tomar otra partida". 25 Para llegar a tener ese nuevo punto de partida es necesario examinar lo que hace posible "mi acceso por la reflexión a un espíritu universal". ${ }^{26}$ Ya Husserl había reconocido que "toda reflexión es eidética y, por lo tanto, deja subsistir irreflejo el problema de nuestro ser y el del mundo". ${ }^{27}$ Es necesario entonces aclarar "el entrelazamiento de mi vida con las otras vidas, de mi cuerpo con las cosas visibles... el recorte de mi campo perceptivo con el de los otros... la mezcla de mi duración con otras duraciones" ${ }^{n}{ }^{28}$ Allí nos encontramos con que "ya no hay filosofia reflexiva sino ...un pensar en círculos... en una relación recíproca". ${ }^{29}$ Esa relación recíproca se llama "apertura al mundo"30 y la "visión bruta" ${ }^{\text {"31 }}$ trata de explicar esta "totalidad que está allá antes de que se sepa cómo y porqué". ${ }^{32} \mathrm{La}$ "apertura al mundo" es la "situación total de que la filosofia debe rendir cuentas". ${ }^{33}$ Esto para la filosofía reflexiva es un problema, porque "retrocede de este lado del mundo", 34 además "para la filosofía reflexiva es una dificultad inextricable comprender cómo una consciencia constitutiva puede poner otra igual a sín. ${ }^{35}$ Se exige entonces una profundización: "una filosofia reflexiva, a menos de ignorarse a sí misma, se ve llevada a interrogarse sobre lo que la precede, sobre nuestro contacto con el ser en nosotros y fuera, antes de toda reflexión". 36

c) La filosofia negativa. ${ }^{37}$ Aquí hay dos clases: la filosofia que piensa verdaderamente la Nada, y la filosofia que concibe al sí mismo "como negatividad y al mundo como positividad". ${ }^{38} \mathrm{La}$ primera "es también una filosofia del Ser", ${ }^{39}$ la segunda es a la que Merleau-Ponty da principalmente atención como tercera posibilidad. Ahora bien, en este segundo sentido, la filosofía es

$$
\begin{aligned}
& { }^{20} \text { p. } 68 . \\
& { }^{21} \text { p. } 67 . \\
& { }^{22} \text { p. } 68 . \\
& { }^{23} \text { p. } 62 . \\
& { }^{24} \text { p. } 69 . \\
& { }^{25} \text { p. } 67 . \\
& { }^{26} \text { p. } 74 .
\end{aligned}
$$

$$
\begin{aligned}
& { }^{27} \text { p. } 71 . \\
& { }_{28} \text { p. } 74 . \\
& { }^{29} \text { pp. } 56-57 . \\
& { }^{30} \text { p. } 57 . \\
& { }^{31} \text { p. } 58 . \\
& { }^{32} \text { p. } 65 . \\
& { }^{33} \text { p. } 74 .
\end{aligned}
$$

$$
\begin{aligned}
& { }^{34} \text { p. } 75 . \\
& { }_{35} \text { p. } 90 . \\
& { }_{36} \text { p. } 104 . \\
& { }_{37} \text { p. } 290 . \\
& { }_{38} \text { p. } 78 . \\
& { }_{39} \text { p. } 80 .
\end{aligned}
$$


negativa como negación de un yo, una negación que nace de la "definición del ipse de adherir a una situación de hecho o de sostenerla como su unión con el Ser" ${ }^{40}$ El "para sí" es pensado como pureza negativa ${ }^{41}$ y mediante él "se busca comprender el hecho de que reconozca semejantes". 42 La subjetividad entonces "es nada y a la vez está en la presencia inmediata del, o en contacto con, el mundo". ${ }^{43}$ Pero ya allí "no hay interacción... no somos del mismo orden. Yo permanezco junto a mi propio centro extraño al ser de las cosas". ${ }^{44}$ Podemos decir que la filosofía negativa presenta como problemas principales desde el punto de vista de la filosofia a que apunta Merleau-Ponty:

I) La absoluta alteridad de la nada y el ser. Aquí rige la fórmula "el Ser es, y la nada no es", 45 así "la nada y el ser son siempre absolutamente otros ${ }^{46}$ y "la intuición del ser es solidaria con algo así como la neguintuición de la nada". ${ }^{47}$ Aquí el problema lo describe Merleau-Ponty de la siguiente manera: en la filosofia del negativo la analítica del ser y de la nada "por principio los opone, los define como exclusivos uno del otro, pero si son opuestos absolutos, no se definen por nada que les sea propio; si uno es negado ya el otro está allí; cada uno de ellos no es sino la exclusión del otro y nada impide, en suma, que intercambien sus papeles: sólo permanece el corte entre ellos; de una y otra parte, aún cuando sean alternativos, componen juntos un solo universo del pensar, ya que cada uno de ellos no es sino su retroceso ante el otro". 48 Ahora bien, "esta apropiación por el pensar de un ser inmediato y de una nada inmediata, esta intuición y esta neguintuición, son el retrato abstracto de una experiencia, y es sobre el terreno de la experiencia donde deben ser discutidas". ${ }^{49}$

II) El otro me mira en mi nada y en mi ser. Si se discute sobre el terreno de la experiencia, la filosofia del negativo presenta la "experiencia de la visión... como aniquilación" ${ }^{150}$ Esto se debe a que "mi negación fundamental no está completa a menos que ella misma no haya sido negada desde fuera y colocada, por una visión extraña, entre el número de los seres" ${ }^{*},{ }^{1}$ y "la experiencia de la mirada de otro sobre mí no hace sino prolongar mi convicción íntima de no ser nada... ${ }^{452}$ De alli que sea "necesario que el otro

$$
\begin{array}{lll}
{ }^{40} \text { pp. } 90-91 . & { }^{45} \text { p. } 120 . & \\
{ }^{41} \text { p. } 100 . & { }^{46} \text { p. } 98 . & \\
{ }^{42} I b i d . & { }^{47} \text { p. } 78 . & \text { pp. } 105-106 . \\
{ }^{43} \text { p. } 104 . & { }^{48} \text { p. } 104 . & 51 \text { p. } 89 . \\
{ }^{44} \text { p. } 78 . & { }^{49} \text { p. } 105 . & { }^{52} I b i d . \\
\hline
\end{array}
$$


sea mi negación o mi destrucción" ${ }^{n 3}$ Sin embargo, ya allí la subjetividad es un "ser solipsista" que es "ya en sí el otro absoluto que él deviene para consigo con la aparición del otro". 54 "Ver es no ser y lo que es visto es el ser" ${ }^{n 5}$ Cuando la subjetividad es nada entonces es una subjetividad que ve; pero cuando el que ve es visto, ese "visionario puro, viene a ser, por una catástrofe ontológica, cosa vista".$^{56}$ La subjetividad que era nada es sorprendida en su ser: por esto "la mirada del otro... me envuelve por completo, ser y nada". 57 Entonces "siento en la superficie de mi ser visible que mi volubilidad se amortigua, que me torno carne, y que al cabo de esta inercia que era yo hay ...un otro que no es una $\operatorname{cosa}^{n} .{ }^{58} \mathrm{El}$ otro significa entonces "una de las formas empíricas del deslizamiento hacia el Ser" ${ }^{.59}$ Viene entonces a hacerse presente el problema de si el otro es realmente mi negación. ${ }^{60}$

III) El otro y la relación. "El otro nace de mi costado por una botadura o un desdoblamiento", 61 "filosóficamente no hay experiencia de otro",62 por esto "una filosofía rigurosa de la neguintuición explica los mundos privados sin encerrarnos allí a nosotros" ${ }^{63} \mathrm{El}$ otro es un desconocido, "no hay una percepción del otro por mi parte; bruscamente mi ubicuidad de visionario es desmentida, me siento visto $\mathrm{y}$ el otro es este $\mathrm{X}$ que está allá, y que necesito pensar bien para explicar el cuerpo visible que de pronto siento tener".64 "La vida del otro, tal como él la vive... es una experiencia prohibida, es un imposible ${ }^{n}{ }^{65}$ Pero allí hay un gran problema: "Si verdaderamente el otro es el otro, es decir, un Para Sí en el sentido fuerte en que yo soy para mí, es necesario que nunca lo sea a mis ojos, es necesario que este otro Para sí nunca caiga bajo mi mirada, es necesario que no haya percepción de otro, es necesario que el otro sea mi negación o mi destrucción" ${ }^{n}{ }^{66}$ Pero "el problema del otro es un caso particular del problema de los otros, la relación con alguien está siempre mediada por la relación con un tercero... si el acceso a otro es entrada a una constelación de otros... es difícil sostener que el otro sea, sin más, la negación absoluta de mí mismon ${ }^{n}{ }^{6}$ Viene por otra parte a establecerse un "sistema de cuatro términos: mi ser para mí, mi ser para otro, el para sí de otro y su ser para mí... hay intersección de mi universo con el de otro". 68

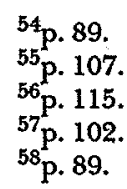

$$
\begin{aligned}
& 59 \text { p. } 101 . \\
& 60 \text { p. } 113 . \\
& 61 \text { p. } 86 . \\
& 62 \text { p. } 100 . \\
& 63 \text { p. } 89 .
\end{aligned}
$$$$
\begin{aligned}
& { }^{64} \text { p. } 119 . \\
& { }^{65} \text { p. } 110 . \\
& { }^{66} \text { p. } 111 . \\
& { }^{67} \text { p. } 113 \text { nota. } \\
& { }^{68} \text { p. } 111 .
\end{aligned}
$$ 
IV) No hay intermundo. Al haber intersección entre mi universo y el de otro se establece "un sistema de perspectivas parciales, referidas a un mismo mundo en el cual coexistimos y donde ellas se recortan ${ }^{n}{ }^{69}$ El otro tiene "el poder de descentrarme, de oponer su centración a la mía" porque somos "dos entradas hacia el mismo Ser $^{n} .{ }^{70}$ En un mundo que "es principal y esencialmente visual ${ }^{71}$ el otro "no puede verme, a mí el invisible, más que porque los dos pertenecemos al mismo sistema del ser para sí y del ser para otro". ${ }^{72}$ En una filosofia rigurosa de la neguintuición "no hay, hablando con propiedad, intermundo". ${ }^{73}$

V) Ambivalencia. "La 'neguintuición' de la nada es la actitud filosófica que pone la reflexión y la espontaneidad en una cierta equivalencia". ${ }^{74}$ Ahora bien, "si una filosofía del negativo es a la vez fijación de esencias y coincidencias con lo vivido, esto no es por azar, inconsecuencia o eclecticismo, sino porque la espontaneidad consiste en ser en el modo de no-ser y la crítica reflexiva [consiste] en no-ser en el modo de ser, y porque estas dos relaciones forman un circuito que somos nosotros" ${ }^{75}$ Pero cuando "la nada y el ser son siempre absolutamente otros... realmente no se unen sino que sólo se suceden más rápido que el pensamiento". ${ }^{76}$ Así la filosofía de la negatividad no se puede asir "justamente porque el nichtiges Nicht no es y el 'hay' se reserva a un ser sin mezcla, positivo y pleno". ${ }^{77}$ Ahora bien, la nada y el ser "son la misma cosa en dos contradictorios=ambivalencia". ${ }^{78}$ Por eso cuando la dialéctica "quiere formularse de una vez por todas, sin resto, enunciarse como doctrina, hacer su propio total ... la negación es llevada al absoluto, viene a ser negación de sí misma... y el movimiento dialéctico deviene identidad pura de los opuestos, ambivalencia",79 allí "el pensar deja de acompañar o de ser el movimiento dialéctico, lo convierte en significación, tesis, o cosa dicha y por ese mismo hecho cae en la imagen ambivalente de la Nada que se sacrifica para que el Ser sea y del Ser que, desde el fondo de su primacía, tolera ser reconocido por la Nada" ${ }^{80} \mathrm{La}$ dialéctica así hablada y no practicada viene a ser "potencia de ser, un principio explicativo", 81 se olvida de ser "pensar de situación, pensar en contacto con el ser... y de ser autocrítica" ${ }^{82}$ y se olvida también de que "toda tesis es idealización" ${ }^{n} .{ }^{83}$

$\begin{array}{lll}{ }^{69} \mathrm{p} .114 . & { }^{74} \mathrm{p} .93 . & { }^{79} \mathrm{p} .127 . \\ { }^{70} \mathrm{Ibid} . & 75 \mathrm{p} .119 . & { }^{80} \mathrm{pp} \cdot 127-128 . \\ { }^{71} \mathrm{p} .115 . & 76 \mathrm{p} .98 . & { }^{81} \mathrm{p} .128 . \\ { }^{72} \mathrm{Ibid} . & { }^{77} \mathrm{p} .119 . & { }^{82} \mathrm{p} .126 . \\ { }^{73} \mathrm{p} .90 . & 78 \mathrm{p} .98 \text { nota. } & { }^{83} \mathrm{p} .129 .\end{array}$


"Al invertir las posiciones de la filosofia reflexiva... la filosofia del negativo se va de paso" 84 por olvidarse de que "por principio, una filosofia del negativo no puede partir de la negación 'pura', ni hacer de ella el agente de su propia negación ${ }^{n} .{ }^{85}$ De esta manera las tres posibilidades, ciencia, filosofía reflexiva y filosofia del negativo, son rechazadas como soluciones al problema del proyecto filosófico. En realidad no sólo la filosofia del negativo sino también las otras dos, hacen "imposible aquella apertura al ser que es la fe perceptivan. ${ }^{86}$

\section{La fe perceptiva}

Hay una "fe que es común al hombre natural y al filosófo desde que abren los ojos" ${ }^{87}$ pero cuando se trata de articularla "se entra a un laberinto de dificultades y de contradicciones" ${ }^{88}$ Se le llama fe "precisamente porque es... una adhesión que se sabe más allá de las pruebas, no necesaria, tejida de incredulidad, amenazada a cada instante por la incredulidad" ${ }^{89}$ Es " $\mathrm{fe}^{n}$ y no "saber", 90 es una "paradoja".91

Esa fe tiene sus exigencias. Las más notables que apunta Merleau-Ponty son cuatro:

a) La percepción "entra en las cosas": 92 "la fe perceptiva... nos aseguraba... acceder a las cosas mismas", 93 la "convicción de ir a las cosas mismas". 94

b) La percepción "se hace de este lado de [mi]... cuerpo":95 "La fe perceptiva... nos aseguraba... llegar a" la percepción "mediante el cuerpo". 96 "La percepción emerge del receso de un cuerpo" .97

c) La fe perceptiva está "convencida de tener que habérselas con una totalidad confusa donde todas las cosas están juntas... y que denomina mundo", 98 tiene la "convicción de que... hay un mundo".99

d) La fe perceptiva expresa "nuestra experiencia... de habitar el mundo por nuestro cuerpo". 100

Sin embargo, aquí existe el problema de que las dos primeras exigencias o convicciones "coexisten sin dificultad" en el uso dia-

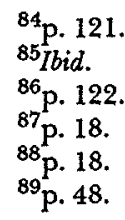

$$
\begin{aligned}
& { }^{90} \text { p. } 49 . \\
& { }_{91} \text { p. } 53 . \\
& 92 \text { p. } 23 . \\
& { }_{93} \text { p. } 51 . \\
& { }_{94}^{94} \text { Ibid. } \\
& { }_{95} \text { p. } 23 .
\end{aligned}
$$$$
\begin{aligned}
& { }^{96} \text { p. } 51 . \\
& { }_{97} \text { p. } 24 . \\
& { }_{98} \text { p. } 91 . \\
& { }_{99} \text { p. } 51 . \\
& { }^{100} \text { p. } 48 .
\end{aligned}
$$ 
rio, pero si son "reducidas a tesis y enunciados, se entre destruyen y nos dejan en la confusión". ${ }^{101}$ La tercera exigencia parece "no abrirnos al mundo sino sellándonos en la serie de nuestros eventos privados". 102

Con la filosofía reflexiva, la percepción viene a ser "el pensamiento de percibir cuando es plena o actual". ${ }^{103}$ También la filosofia negativa "hace imposible esta apertura al ser que es la fe perceptiva". 104 De aquí que Merleau-Ponty declare igualmente incompletas ambas filosofias: "No hay apertura al ser para una filosofia del pensamiento y de nuestros pensamientos inmanentes, pero tampoco la hay para una filosofía de la nada y del ser, porque tanto en un caso como en el otro, el ser es lejano, a distancia". 105

"Es necesario comprender la percepción como... pensamiento interrogativo que deja ser al mundo percibido más que posarlo, delante del cual las cosas se hacen y se deshacen en algo así como un resbalo, de este lado del sí y del no". ${ }^{106} \mathrm{La}$ fe perceptiva es "todo aquello que se ofrece al hombre natural en original dentro de una experiencia-origen con el vigor de lo que es inaugural y presente en persona según una vista que es para él última y que no sabría concebirse más perfecta o más próxima... ${ }^{107}$ Lo que se da en esa experiencia-origen es "la experiencia del ser bruto". ${ }^{108}$

\section{El ser bruto}

Sin embargo, respecto a esa experiencia que es fe perceptiva, ${ }^{109}$ ¿cuál es la misión de la filosofía? Hablando en términos generales podemos decir que Merleau-Ponty después de criticar la filosofia como fijación de esencias, da tres características de lo que él considera la filosofía y en ellas delínea la misión de la filosofia. Las tres son parte de una sola visión de la filosofía. Pero antes vamos primero a ver un poco de lo que nos dice acerca de la fijación de esencias.

La fijación de esencias como búsqueda del quid sit viene a ser tal cuando la "cuestión común de an sit"110 no desemboca en ninguna parte. Pero "por la cuestión quid sit, más bien que por la duda, la filosofia logra desprenderse de todos los seres porque les cambia el sentidon. ${ }^{111}$ El cambio de sentido les viene del hecho

$$
\begin{aligned}
& { }^{101} \text { pp. } 23-24 . \\
& { }^{102} \text { p. } 51 . \\
& { }^{108} \text { p. } 50 . \\
& { }^{104} \text { p. } 122 .
\end{aligned}
$$$$
\begin{aligned}
& { }^{105} \text { p. } 122 . \\
& { }^{106} \text { p. } 138 . \\
& { }^{107} \text { pp. } 209-210 . \\
& { }^{108} \text { p. } 209 .
\end{aligned}
$$

${ }^{109}$ Ibid.

${ }_{110}^{11}$ p. 144. 
que "las esencias son este sentido intrínseco... (que es) el sistema de todo lo que es posible ante la mirada del espectador puro". 112 El espectador puro lee el sentido y "la filosofía vendría a ser esta misma lectura del sentido llevada a su término, ciencia exacta, la única exacta". ${ }^{113}$ Pero "la esencia no es $l a$ respuesta a la cuestión filosófica, la cuestión filosófica no la hace en nosotros un espectador puro". ${ }^{114}$ La fijación de esencias no es la cuestión última por tres razones: I) La esencia es un "in-variante" y la "esencialidad de la esencia es medida exactamente por el poder que tenemos de hacer variar la cosa. Una esencia pura... no puede resultar más que de un ensayo de variación total". 115 II) Una esencia pura "exigiría un espectador mismo sin secretos, sin latencia",116 pero al mismo tiempo "tendríamos que tomar tal distancia respecto a ella que la pusiera toda entera bajo nuestra mirada" 117 y entonces quizá ya no sería experiencia. III) Hay un saber "que está por debajo de la esencia": el saber que responde a la pregunta; "¿đde dónde tenemos la hipóteis, de dónde sabemos que hay alguna cosa, que hay un mundo ? $^{118}$ Ese saber "es la experiencia de la cual la esencia forma parte y a la cual no envuelve ${ }^{n} .119$ Por esto "sería un error definir la filosofía como búsqueda de esencias o como fusión con las cosas y los dos errores no son muy diferentes". 120

Las tres características de la filosofia en la visión de MerleauPonty son:

a) Interrogación sin presuposiciones. ${ }^{121}$ Debe por tanto quitar las verdades establecidas ${ }^{122}$ y vencer la tentación de "construir la percepción a partir de lo percibido" y de "construir nuestro contacto con el mundo a partir de lo que este contacto nos ha enseñado sobre el mundon. ${ }^{123}$ Tampoco debe introducir en la descripción "conceptos salidos de la reflexión" ${ }^{n}{ }^{124}$ Por esto mismo no es una simple espera de una significación que vendría a colmarla"; 125 por el contrario, "es propio de la interrogación filosófica retornar sobre sí misma, preguntarse qué es preguntar y qué es responder. Esta pregunta al cuadrado, una vez hecha, no se borra jamás". ${ }^{126}$ Allí se puede ver "la interrogación como rela-

$\begin{array}{lll}{ }^{112} \text { Ibid. } & 117 \text { pp. } 149-150 . & 122 \mathrm{p} .207 . \\ 113 \text { p. } 144 . & 118 \text { p. } 147 . & 123 \text { Ibid. } \\ 114 \text { p. } 147 . & 119 \text { Ibid. } & 124 \text { p. } 209 . \\ 115 \text { p. } 149 . & 120 \text { pp. } 168-169 . & 125_{\text {p. }} 160 . \\ 116 \text { Ibid. } & 121 \text { p. } 207,314 . & 126 \text { Ibid. }\end{array}$


ción última con el Ser y como órgano ontológico". ${ }^{127}$ La filosofía como interrogación, "no puede consistir sino en mostrar cómo el mundo se articula a partir de un cero de ser que no es nada, es decir, en instalarse sobre el bordo del ser, ni en el para Sí ni en el en Sí, en la juntura, allí donde se cruzan las múltiples entradas del mundo". ${ }^{128}$ No presupone "ninguna de las 'condiciones' fisicas o fisiológicas que limitan la percepción para el sabio", "ninguno de los postulados de una filosofia sensualista o empírica", y finalmente "ninguna definición de una 'primera capa' de experiencia". ${ }^{129} \mathrm{La}$ filosofía es una interrogación que es "cuestión-saber que por principio no puede ser sobrepasada por ningún enunciado o 'respuesta', y por tanto, quizá el modo apropiado de nuestra relación con el Ser... que... está silenciosamente detrás de todas nuestras afirmaciones, negaciones, y aun detrás de todas las cuestiones formuladas..." ${ }^{130}$ Lo único que admite esta interrogación sin presuposiciones, es lo que ella ha visto "surgir de nuestra experiencia del ser bruto". 131

b) Retorno a la fe perceptiva. "La percepción como encuentro con las cosas naturales está en el primer plano de nuestra investigación... como arquetipo del encuentro original: imitado y renovado en el encuentro con el pasado, lo imaginario, la idea. Qué será nuestra interrogación misma y nuestro método, no lo sabemos desde antes". ${ }^{132}$ Este retorno a la fe perceptiva nos presenta dos características: I) la filosofía es "reconquista del ser bruto o salvaje" 133 y el ser bruto es "objeto de la filosofia"; ${ }^{134}$ II) "la filosofía es la fe perceptiva interrogándose sobre sí misman ${ }^{n}{ }^{135}$

- c) Construcción humana que lleva al habla. "El Ser es aquello que exige creación de nuestra parte para poder tener su experiencia"136 y "la filosofía, precisamente como 'Ser que habla en nosotros', expresión de la experiencia muda de por sí, es creaciónn". ${ }^{137}$ Pero "se trata de una creación evocada y engendrada por el Lebenswelt como historicidad operante, latente, que la prolonga y da testimonio de ella". ${ }^{138}$ Sin embargo, el "paso del mundo mudo al mundo hablante ${ }^{n 139}$ sólo se efectúa mediante la "palabra operante $^{n 140}$ y es reversible con el silencio ${ }^{141}$ y con el significado y la significación. ${ }^{142}$ Sólo en esa reversibilidad se encuentra la unión de

$$
\begin{array}{lll}
{ }^{127} \text { p. } 162 . & 133 \text { p. } 139 . & \\
{ }^{128} \text { p. } 314 . & 134 \text { p. } 138 . & \\
129 \text { p. } 209 . & 135 \text { p. } 139 . & { }^{139} \text { p. } 202 . \\
130 \text { p. } 171 . & 136 \text { p. } 251 . & { }^{140} \text { lbid. } \\
{ }^{131} \text { p. } 209 . & 137 \text { p. } 250 . & 141 \text { p. } 171 . \\
{ }_{132} \text { p. } 210 . & 138 \text { p. } 228 . & { }^{142} \text { p. } 202 .
\end{array}
$$


los puntos de vista de Husserl (filosofia como restitución del poder de significación) y de Paul Valéry (el lenguaje, como voz del mundo, no necesita restitución de su poder de significación), y esta reversibilidad viene a ser una "verdad última". ${ }^{143}$ Sólo la "lógica en acciónn"144 es capaz de seguir esta reversibilidad y llevar al habla al mundo mudo; ese llevar al habla es la dialéctica. Por esto Merleau-Ponty nos dice, "es necesario sustituir la intuición del ser y la neguintuición de la nada por una dialéctica ... el pensar dialéctico es aquél que admite relaciones recíprocas o interacciones... las implica en el movimiento y no las sobrevuela". ${ }^{145}$

De esta manera tenemos una visión del proyecto y misión de la filosofia que Merleau-Ponty nos presenta. Vamos ahora a ver el segundo punto: su realización.

\section{B. REALIZACIÓN}

Giertamente no podemos decir que en Visible e Invisible tengamos ya una realización de la filosofia proyectada; por el contrario, como nos lo muestra el estado del manuscrito y las Notas de Trabajo, el escrito de Merleau-Ponty nos abre avenidas que no recorre, apunta a un horizonte al cual apenas si entra. Nos queda entonces abierta la posibilidad de organizar una manera de realizar y presentar algunos problemas a los cuales aún no ofrece solución. Por esto lo que se escribe en esta segunda parte es un esquema de una posibilidad entre otras muchas.

\section{La dialéctica: hiperdialéctica. ${ }^{1}$}

a) Sus características. En cursos de los jueves (1955-56), "La filosofía dialéctica", ya Merleau-Ponty había resumido algunas de las definiciones de la dialéctica:

El pensar dialéctico ha sido definido. 1) Como pensar de contradictorios. Es decir, que no admite entre ellos ni la conciliación relativista, ni aquella identidad por equívoco sobre la que juega la 'mala dialéctica'... La noción hegeliana de negación de la negación... es la fórmula de toda contradicción operante... es el pensar dialéctico mismo, como fecundidad de la contradicción... Nosotros lo hemos acercado a la noción moderna de la

${ }^{143}$ pp. $203-204$.
${ }^{144}$ p. 137.

${ }^{145}$ p. 123.

${ }^{1}$ p. 129. 
trascendencia, es decir, de un ser por principio distante, frente al cual la distancia es una lazo, y con el cual no se tendría coincidencia en esa distancia. Aquí y allá, la relación de sí para consigo pasa por fuera, el inmediato exige la mediación, o aún más, hay una mediación por sí"... 2) Como pensar 'subjetivo'. El pensar dialéctico se desarrolló antes de la filosofía reflexiva y... toma como problema su propio comienzo... Se puede decir, sin embargo, que la dialéctica es pensar 'subjetivo' en el sentido que Kierkegaard o Heidegger han dado a la palabra: no hace reposar al Ser sobre sí mismo sino que lo hace aparecer ante alguien, como respuesta a una interrogación... En lo que tiene de más negativo la subjetividad tiene necesidad de un mundo y en lo que tiene de más positivo el ser tiene necesidad de un no-ser que le circunscriba y determine. Por esto, el pensar dialéctico invita a una revisión de las nociones ordinarias de sujeto y objeto"... 3) Como pensar circular. Porque no quiere sacrificar ni lo irreflexionado ni lo reflexionado, uno al otro, el pensar dialéctico parece ante sus propios ojos como desarrollo al mismo tiempo que como destrucción, de lo que era antes que ella, e igualmente sus conclusiones guardan en sí mismas todo el progreso que ha llevado hasta allí. La conclusión no es realmente sino la integración de los pasos anteriores. El dialéctico es siempre un 'emprendedor' [commençant $]$... la dialéctica ha sido siempre una experiencia del pensar, es decir, un caminar en el curso del cual aprende, aun cuando lo que aprende haya ya estado allí, 'en sí, antes que ella y que la dialéctica no sea sino su paso al ser para sí...2

Luego la caracteriza como pensar negativo. ${ }^{3}$ En los cursos de los lunes (1955-56), "Textos y comentarios sobre la dialéctica", nos dice: "La leyenda de Zenón nos muestra el paso de un pensar que denuncia escándalos lógicos en nombre del ideal de la identidad, a un pensar que por el contrario acoge la contradiccion como movimiento del ser; de una dialéctica habladora y 'ventrílocua' a la verdadera dialéctica". ${ }^{4}$ Podemos decir que las tres primeras características las conserva Merleau-Ponty como características de su propia dialéctica, hasta cierto punto. ${ }^{5}$ Así nos habla de un "pensar en círculo", 6 un pensar en el que reflexión e irreflexión están en "relación recíproca... y en el que el fin está

${ }^{2} \mathrm{M}$. Merleau-Ponty, Résumés de Cours, NRF, Ga-

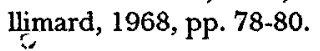

${ }^{3}$ Résumes..., p. 82.

${ }^{4}$ Résumés..., pp. 85-86.

${ }^{5}$ Le visible et... cfr. pp.
$120,124-126$.

6 p. 56.

7 p. 57. 
en el comienzo y el comienzo está en el fin". ${ }^{7}$ Hay una cuarta característica que Merleau-Ponty apunta en Visible e Invisible cuando nos habla de la "dialéctica como pensar de situación, pensar en contacto con el ser". ${ }^{8}$ Respecto a las características de la dialéctica el pensamiento de Merleau-Ponty no apunta mucho cambio en las Notas de Trabajo y allí no vemos prácticamente ninguna referencia que le añada algo nuevo. Sin embargo, como vimos al final de la sección anterior, la filosofía lleva al habla el mundo mudo dentro de la dialéctica. Una dialéctica tal que "si se quiere guardar su espíritu, es necesario, ni siquiera nombrarla". ${ }^{n}$ Esta puede ser considerada como una quinta característica de la dialéctica de Merleau-Ponty. Por esto la dialéctica está ligada al lenguaje.

b) El lenguaje. La tarea del filósofo no es un trabajo de escribano que "se queda en trabajo de lenguaje más que de 'pensamiento'..." ${ }^{10}$ Por esto en repetidas ocasiones Merleau-Ponty habla del silencio del filósofo ${ }^{11}$ y nos dice "el filósofo habla, pero esto en él es una debilidad, una debilidad inexplicable: debería callarse, coincidir en silencio, y unirse dentro del Ser con una filosofía que ya allí está hecha". 12 Por esto, si habla tiene que ser "un lenguaje de coincidencia, una manera de hacer hablar a las cosas mismas". ${ }^{13}$ Este hacer hablar a las cosas mismas, el habla de las cosas mudas es el logos. ${ }^{14}$ Este logos es al mismo tiempo silencio, 15 es lenguaje operante, ${ }^{16}$ es lenguaje-cosa, ${ }^{17}$ es habla de las cosas mudas, ${ }^{18}$ es palabra pensante, ${ }^{19}$ es realización en el hombre, ${ }^{20}$ es igualmente Ser bruto ${ }^{21}$ y fe perceptiva. ${ }^{22}$ Por esto, como se verá después, la filosofía de Merleau-Ponty "se tiene... en el cruce de las avenidas".23

\section{La nueva ontología: ontologia perceptiva}

La ontología de Merleau-Ponty es fe perceptiva, es una "apertura al ser"24 que mantiene al Ser bruto como su horizonte, ${ }^{25}$ es una "búsqueda de la visión 'salvaje' del mundo"26 y es dialéctica. De esta manera nos dice: "Nuestro punto de partida... será...: hay

\begin{tabular}{|c|c|c|}
\hline $\begin{array}{l}{ }^{7} \mathrm{p} .57 . \\
{ }^{8} \mathrm{p} .126 . \\
9 \mathrm{p} .126 . \\
{ }^{10} \text { Resumés, cursos de } \\
1952-53, \text { p. } 40 . \\
{ }^{11} \text { Cfr. Résumes, cursos de } \\
1958-59, \text { p. } 156 . \\
{ }^{12} \text { Le visible et...,p. } 166 .\end{array}$ & $\begin{array}{l}{ }^{13} \mathrm{p} .167 . \\
14 \mathrm{p} .168 . \\
15 \text { p. } 171,230 . \\
{ }^{16} \text { pp. } 201-202 . \\
17 \text { p. } 168 . \\
18 \text { Ibid. } \\
{ }^{19} \text { p. } 229 . \\
{ }^{20} \text { p. } 328 .\end{array}$ & $\begin{array}{l}{ }^{21} \text { p. } 264 . \\
{ }^{22} \text { Cfr. pp. } 223-224 . \\
{ }^{23} \text { p. } 212 . \\
{ }^{24} \text { Cfr. p. } 77 . \\
{ }^{25} \text { p. } 136 ; \text { cfr. p. } 250 . \\
{ }^{26} \text { p. } 236 .\end{array}$ \\
\hline
\end{tabular}


ser, hay mundo, hay algo; en el sentido fuerte en que el griego utiliza to legein, hay cohesión, hay sentido". ${ }^{27}$ Por esto vamos primeramente a analizar algunas nociones fundamentales para luego ver los dos elementos principales de esa ontología.

I) Lebenswelt. "Hacemos una filosofía del Lebenswelt, nuestra construcción (en el modo de la 'lógica') nos hace reencontrar este mundo del silencio":28 "El Lebenswelt como historicidad operante ${ }^{n}{ }^{29}$ Pero hay una indicación de que Merleau-Ponty pensaba en parte modificar esta visión, ya que nos dice que "el Lebenswelt es subjetivo". ${ }^{30}$ Por eso el programa de "desvelamiento del Ser salvaje o bruto por el camino de Husserl y del Lebenswelt al cual se llega" queda todavía como problema, ${ }^{31}$ porque "la reconquista del Lebenswelt es la reconquista de una dimensión dentro de la cual las objetivaciones de la ciencia guardan ellas mismas un sentido y dentro de la cual deben ser comprendidas como verdaderas..." ${ }^{12}$ Frente al Lebenswelt, Merleau-Ponty nos habla del "mundo perceptivo" en el sentido del Ser en Heidegger, ${ }^{33}$ un ser como "Wesen (verbal)".${ }^{34}$ Cuando ese mundo es interrogado, "no nos preguntamos si el mundo existe, sino que nos preguntamos qué sea para el mundo existir" ${ }^{35}$ Por esto "la filosofia no es ruptura con el mundo, no es coincidencia con él, pero tampoco es alternancia entre ruptura y coincidencia... Nos ha parecido que la tarea era describir estrictamente nuestra relación con el mundo... como simple apertura; es por la apertura por lo que podemos comprender el ser y la nada". 36 "La filosofia no busca pues analizar nuestra relación con el mundo, deshacerla...; pero tampoco se termina con una constatación inmediata y global del Ser..." ${ }^{n 7}$

II) Ser. "Lo que finalmente amerita el nombre de ser... es... el sistema de perspectivas que introduce [en el horizonte del ser 'puro'] que el ser integral es...", 38 pero este sistema de perspectivas tiene como puntos de referencia los dos elementos principales de la ontología de Merleau-Ponty que veremos más adelante. Es un "ser de indivisión", principio de la ontología, y puede describirse como un "espacio topológico". 39

III) Cosa. En el "Anexo" nos habla Merleau-Ponty de "la cosa y alguna cosa". ${ }^{40}$ "Si buscamos qué quiere decir para nosotros 'la

${ }^{27}$ p. 121
28 p. 224.
29 p. 228.
${ }^{30}$ p. 239.
${ }^{31}$ p. 237.
${ }^{32}$ p. 236.

${ }^{33}$ p. 223.

34 p. 228.

${ }^{39}$ p. 262, 264.

35 p. 130.

${ }^{36}$ p. 135

${ }^{37}$ p. 136.

${ }^{38}$ p. 111.

${ }^{40}$ Cfr. p. 212

Sckart; esse y "esse hoc,scilicet lapis",en Porl in Op. Prop.

3, pp. 166-167. 
cosa', encontramos que es lo que reposa en sí mismo, que es exactamente lo que es, todo en acto, sin ninguna virtualidad ni potencia, que es por definición 'trascendente', fuera, absolutamente extraño a toda interioridad".41 Pero la cosa "es un nudo de propiedades... principio de identidad". ${ }^{42}$ Por esto "la identidad de la cosa misma, ese cierto asiento propio, de reposo en sí misma, esta plenitud y esta positividad que le hemos reconocido, exceden ya la experiencia, son ya una interpretación segunda de la experiencia". ${ }^{43}$

IV) Sujeto. Antes de ser "espectador puro",44 "soy un campo de experiencias donde se dibujan solamente la familia de las cosas materiales y otras familias, y el mundo como su estilo común; la familia de las cosas dichas y el mundo de la palabra como su estilo abstracto y descarnado de alguna cosa en general" ${ }^{45} \mathrm{El} \mathrm{sujeto}$ verdadero es un "sujeto percipiente, como Estar tácito, silencioso..."46 y es "sujeto hablante: ...sujeto de una praxis". $47 \mathrm{El}$ verdadero sujeto es Topológico: es "campo...sistema jerarquizado de estructuras abiertas por un hay inaugural".48

Vamos ahora a ver los dos conceptos fundamentales de esa ontología que tiene como horizonte al Ser bruto o salvaje; el "terreno extraño al que la interrogación propiamente dicha nos da acceso" ${ }^{49}$ Estos dos conceptos fundamentales son:

a) El inter como reversibilidad. Podríamos quizá decir que la reversibilidad se basa en "la pertenencia de cada experiencia al mismo mundo..., a título de posibilidades del mismo mundo",50 "variantes del mismo mundo", 51 y difiere de la "reversión dialéctica" ${ }^{n 2}$ Sólo de esta manera se está en posición de descubrir el "entrelazamiento de mi vida con las demás, de mi cuerpo con las cosas visibles, ...el recorte de mi campo perceptivo con el de otros, ...la mezcla de mi duración con las otras duraciones ${ }^{n} .53$ Este inter es "enrollamiento", 54 es una inserción en la dehiscencia, ${ }^{55}$ es empotramiento, ${ }^{56}$ es crucero, ${ }^{57}$ es círculo, ${ }^{58}$ es reciprocación, ${ }^{59}$ es cohesión, ${ }^{60}$ es "como", 61 es lazo, ${ }^{62}$ es juntura, 63 es "nudo", ${ }^{64}$ por

$\begin{array}{lll}{ }^{41} \text { p. } 77 . & { }^{50} \text { p. } 65 . & \\ { }^{42} \text { p. } 214 . & { }^{51} \text { p. } 65 . & \\ { }^{43} I b i d . & { }^{52} \text { p. } 204 . & { }^{60} \text { p. } 183 . \\ { }^{44} \text { p. } 148 . & 53 \text { p. } 74 . & { }^{61} \text { Vgr. 'la carne } \\ { }^{45} \text { p. } 149 . & 54 \text { p. } 191 . & \text { como expresion", } \\ { }^{46} \text { p. } 254 . & 55 \text { p. } 201 . & \text { p. } 190 . \\ { }^{47} \text { p. } 255 . & { }^{56} \text { p. } 227 . & { }^{\text {p. }} 195 . \\ { }^{48} \text { p. } 292 . & 57 \text { p. } 212 . & { }^{64} \text { p. } 314 . \\ { }^{49} \text { p. } 185 . & { }^{58} \text { p. } 188 . & \end{array}$


eso Merleau-Ponty le llama "entrelazamiento".65 Esta reversibilidad puede verse principalmente en tres aspectos:

I) Intercorporeidad. Son apenas algunas indicaciones las que tenemos de ella ${ }^{66}$ Nos habla del campo que podría abrirse "para una 'intercorporeidad" 67 y que haría posible hacer con otros "la experiencia de un solo cuerpo ante un solo mundo..." 68 "Con la reversibilidad... lo que se nos abre es ...un mundo intercorporal..." 69 La intercorporeidad es el funcionar "con un cuerpo único", ${ }^{70}$ es "transitividad de un cuerpo a otro",71 es decir, la "intercorporeidad en cuanto natural". ${ }^{72}$

II) Intermundo. Merleau-Ponty hace notar que "en una filosofia rigurosa de la neguintuición... no hay, propiamente hablando, intermundo". ${ }^{73}$ "El mundo sensible y el mundo histórico son siempre intermundos, ya que son aquello que, más allá de nuestras visiones, las hacen solidarias entre ellas y solidarias de las de los demás, [ya que son] las instancias a las que nos dirigimos desde el comienzo de nuestro vivir, [son] los registros donde se inscribe lo que vemos, para devenir allí cosa, mundo, historia". ${ }^{74}$ El intermundo como mundo percibido, "está más acá o más allá de la antinomia" sujeto-objeto. ${ }^{75}$

III) La carne. Este punto lo veremos después.

Sería quizás posible incluir también la noción de intersubjetividad pero no en el sentido husserliano, sino con una idea de la subjetividad, ${ }^{76}$ en la que la díada yo-otro es una "fórmula insuficiente". ${ }^{n 7}$

b) El cruce como reversibilidad nunca realizada. La reversibilidad es "siempre inminente y jamás realizada de hecho", ${ }^{78}$ es un "robo incesante",79 un "usurpamiento", 80 el "pliegue", 81 una separación, ${ }^{82}$ es distanciamiento, ${ }^{83}$ es "movida", 84 un hiatus, ${ }^{85}$ abismo, ${ }^{86}$ es "trascendencia" como "identidad en la diferencia". ${ }^{87}$ Así nos dice, "nos paramos, como el hombre natural, en nosotros $y$ en lás cosas, en nosotros y en otro, en el punto donde por una especie de cruce, devenimos los otros y devenimos mundo". 88 "La

\footnotetext{
${ }^{65} \mathrm{p} .182$.

${ }^{66}$ p. 226.

${ }^{67}$ p. 185.

${ }^{68}$ p. 186.

${ }^{69} \mathrm{p} .188$.

${ }^{70}$ p. 268.

71 p. 188.

72 p. 222.

${ }^{73}$ pp. 89-90.
}

$$
\begin{array}{ll}
{ }^{74} \text { p. } 116 . & \\
75 \text { p. } 41 . & \\
76 \text { p. } 322 . & \\
77 \text { p. } 274 \text {; cfr. nota p. } 113 . & { }^{82} \text { p. } 194,268 . \\
{ }^{78} \text { p. } 194 . & { }^{84} \text { p. } 194 . \\
79 \text { Ibid. } & { }^{85} \text { p. } 195 . \\
{ }^{80} \text { p. } 292 . & 86 \text { p. } 180 . \\
{ }^{81} \text { p. } 317 . & 87 \text { p. } 279 . \\
{ }^{83} \text { p. } 166 . & 88 \text { p. } 212 .
\end{array}
$$


verdadera filosofia... es asir este cruce ${ }^{\prime \prime}{ }^{89}$ Como podemos ver en las Notas de Trabajo, este punto quizás iba a ser tratado con mayor amplitud: "el cruce",90 "el cruce y reversibilidad", 91 "tiempo y cruce" ${ }^{\prime 2}$ En el cruce encontramos varios conceptos que vamos a ver a continuación:

I) La nada como diferencia de idénticos. ${ }^{93}$

II) Exterioridad-interioridad. Merleau-Ponty nos habla en repetidas ocasiones del aspecto exterior o "envoltura exterior ${ }^{n 94} y$ del aspecto interior o "armadura interior"; 95 nos habla de "los horizontes exteriores e interiores". 96 Por otra parte, utiliza repetidas veces el concepto del Ineinander ${ }^{97}$ o inmanencia mutua de Husserl. ${ }^{98}$ Horizonte exterior e interior se los atribuye a Husserl $^{99}$ y es muy posible que en el futuro los hubiera considerado en el sentido de la sección "Tiempo y cruce". 100

III) Para-Sí Para Otro-En Sí. "La integración en sí-por sí, se hace, no en la consciencia absoluta sino en el Ser de promiscuidad". ${ }^{101}$ Sólo por la reversibilidad "hay paso del 'Para Si' al Para Otro ....No hay Para Sí y Para Otro. Son el otro lado respecto al otro". ${ }^{102}$ La dicotomía Para Sí y Para Otro viene de la filosofia de la visión, ${ }^{103}$ y "la verdad del En Sí-Para Sí sartreano es la intuición del Ser puro y la neguintuición de la Nada" ${ }^{104}$ Pero MerleauPonty nos dice: "Tomo mi punto de partida allí donde Sartre hizo su punto de llegada, en el Ser retomado por el Para-Sín ${ }^{105}$

IV) Visible-Invisible. Merleau-Ponty se propuso ir más allá de la filosofía de la visión. ${ }^{106}$ De allí que tomar simplemente visible e invisible como una dualidad ${ }^{107}$ no resuelta, sea una equivocación. ${ }^{108}$ Más bien, debe tomarse en el contexto del inter y el cruce. El hecho de que en las Notas de Trabajo este tema aparezca numerosas veces no sólo nos apunta al título del libro ${ }^{09}$ sino principalmente a una reconsideración por parte de Merleau-Pontydel cruce y del inter para visible-invisible. Quizás podemos decir que esto se debe a que el tema está íntimamente ligado con el de cuerpo-carne que veremos a continuación. "Lo visible es...presen-

\footnotetext{
${ }^{89}$ p. 252.

90 p. 268.

91 pp. 316-319.

${ }_{92}^{9}$ p. 321.

93 p. 316 .

${ }_{94}$ p. 194.

${ }^{95}$ p. 195.

96 p. 173.
97 p. 226.
}

$$
\begin{aligned}
& { }^{98} \mathrm{Cfr} . \text { Résumés, cursos } \\
& 1958-59 \text {, "Posibilidad } \\
& \text { de la filosofia". } \\
& { }_{99} \text { p. } 195 . \\
& { }_{100} \text { p. } 321 . \\
& { }^{101} \text { p. } 307 . \\
& { }^{102} \text { p. } 317 . \\
& { }^{103} \text { pp. } 111-112 . \\
& { }^{104} \text { p. } 118 .
\end{aligned}
$$

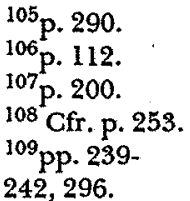


tación original de lo no presente originalmente... lo visible deja de ser un inaccesible si lo concibo, no según un pensar proximativo, sino como englobando, revestimiento lateral, carne". ${ }^{110}$ Lo invisible no es "otro visible 'posible', o un 'posible' visible para otro"; 111 "lo invisble está allísin ser objeto, es la trascendencia pura, sin máscara óntica. Y los 'visibles' mismos también, a fin de cuentas, no están ellos mismos sino centrados sobre una red de ausencias". 112 "Todo visible es un invisible ${ }^{n 113}$ no en el "sentido de una contradicción"114 sino que "hay que comprender que la visibilidad misma comporta una no-visibilidad... Lo invisible de lo visible. Ésta es su pertenencia a un rayo del mundo". ${ }^{115}$ La relación mundo-ser es la relación de "visible e invisible (la latencia), lo invisible no es otro visible ('posible' en sentido lógico), un positivo meramente ausente" ${ }^{116}$ El invisible no es "una negación en sentido logico" o mero resultado del hecho de que "lo visible ha sido definido como dimensionalidad del Ser", 117 no es resultado del "error filosófico total que es creer que lo visible es presencia objetiva (o idea de esta presencia)..."118 Por esto el vidente está "en cruce con" lo visible. 119

Ciertamente los dos elementos principales de la ontología de Merleau-Ponty, inter y cruce vienen a apuntarnos a un camino de realización de su proyecto filosófico. ${ }^{120}$ Antes de pasar al punto que aquí tomaremos como central en la presentación, es necesario notar que Merleau-Ponty en sus escritos nos da dos posibilidades, diferentes hasta cierto punto, para la contraparte del inter: nos da a veces "chiasma", 121 cruce, y a veces "chiasme", 122 que aunque tienen una sola palabra griega como origen (xiasma), en francés tienen dos usos un tanto diferentes, aun cuando las dos palabras signifiquen un cruzamiento.

\section{La came}

"No hay nombre en la filosofia tradicional para designar" ésta. ${ }^{123}$ "Lo que llamamos carne no tiene nombre en ninguna filosofia" ${ }^{124}$ Por éstas y otras indicaciones, parecería que Merleau-Ponty apunta a la nueva visión que abre su ontología mediante las

${ }^{110}$ p. $270 ;$ cfr. 272.

$281,286$.

111 p. 282.

112 p. 283.

113 p. 300.

${ }^{114}$ Ibid.

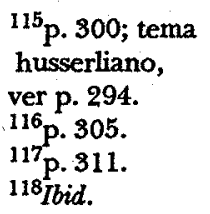


dos características antes vistas. De allí que tenga que ser puesta como "un elemento... (en el sentido de cosa general) del Ser". ${ }^{25}$

Pero cuando nos dice que "la reversibilidad que define a la carne existe en otros cuerpos"126 y también al referirse a "este primer esbozon, 127 parece darnos una idea diferente dẹ la carne.

Por esta razón vamos aquí a ver el punto de unión de lo visible y lo invisible, ${ }^{128}$ que es carne del mundo, ${ }^{129}$ carne de $\operatorname{cosas}^{130} \mathrm{y}$ carne nuestra. ${ }^{131}$

a) Carne del mundo. "La carne del mundo es el Ser-visto, i.e., un Ser que es preeminentemente percipi, y por el cual se puede comprender el percipere ${ }^{n}{ }^{132} \mathrm{El}$ mundo visto es la nueva definición del vidente. ${ }^{133}$ "La carne del mundo, (el 'quale') es indivisión de este Ser sensible que soy yo, y de todo lo demás que se siente en mi..." ${ }^{\text {134 }}$ Esta carne no debe ser pensada "a partir de las substancias -cuerpo y espíritu, porque entonces sería unión de contradictorios", ${ }^{135}$ sino como una "textura". ${ }^{136}$ No es ni substancia, ni materia, ni representación, ni espíritu. ${ }^{137}$

La came es "enrollamiento de lo visible sobre el cuerpo vidente, de lo tangible sobre el cuerpo tocante". ${ }^{138}$ Es una Visibilidad, una generalidad de lo Sensible en sí, es anonimato innato del Mímismo, ${ }^{139}$ es punto donde visible y vidente se reciprocan, donde ya no se sabe ni quién ve ni quién es visto, ${ }^{140}$ es el "medio formador del sujeto y el objeto", 141 es un "elemento... del Ser", una "facticidad, lo que hace que el hecho sea hecho", 142 "es una noción última... pensable por sí misma". 143 "La carne como expresión... (es) punto de inserción del hablar y el pensar en el mundo del silencio". 144

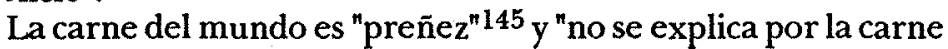
del cuerpo" sino que "es por la carne del mundo por lo que se puede a fin'de cuentas comprender el propio cuerpo" ${ }^{146}$ Sin embargo, "mi cuerpo está hecho de la misma carne que el mundo"147 y "esta carne de mi cuerpo es participada por el mundo... mi cuerpo... es el punto nulo de todas las dimensiones del cuerpo" ${ }^{148}$ Por esto "la carne de las cosas ya nos habla de nuestra carne, y de la

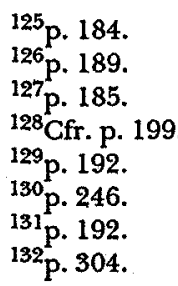

$$
\begin{aligned}
& { }^{133} \text { p. } 108 . \\
& { }^{134} \text { p. } 309 . \\
& 135 \text { p. } 193 . \\
& { }^{136} \text { p. } 192 . \\
& { }^{137} \text { pp. } 183-184 \\
& { }^{138} \text { p. } 191 . \\
& { }^{139} \text { p. } 183 . \\
& { }^{140} \text { Ibid. }
\end{aligned}
$$

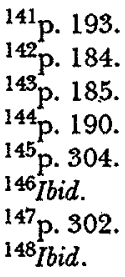


del otro", 149 y "la Tierra como arché primordial pone en evidencia la historia primordial carnal". ${ }^{150}$

b) Nuestra carne. Nuestra carne es "emblema concreto de una manera de ser general", 151 por esto no puede sin más tomarse como "cuerpo objetivo". ${ }^{152}$ Por esto mismo, "esta carne que se ve y que se toca no es toda la carne, ni esta corporeidad masiva, todo el cuerpo". ${ }^{153}$ Ahora bien, "cuando hablamos de la carne de lo visible... queremos decir... que el ser carnal que tiene varias hojas o varias caras, ser de latencia y presentación de una cierta ausencia, es un prototipo del Ser, del cual nuestro cuerpo, el sintiente sensible, es una notable variante... ${ }^{\text {154 }}$ Aquí tenemos el paso de la carne en general a nuestro cuerpo. Por esto la carne es también "Leib". ${ }^{155}$ La carne como "carne de nuestro cuerpo"156 nos muestra en escala, en una cierta dimensión, ${ }^{157}$ esa "manera de ser general $^{158}$ a la que apunta Merleau-Ponty.

En la dimensión del cuerpo estesiológico, cuerpo de cinco sentidos $^{159}$ se retoman los puntos principales de la filosofía de Merleau-Ponty: la percepción (cfr. fe perceptiva), el habla, la reversibilidad y el cruce.

La percepción no es ni "el cuerpo en el mundo y el vidente en el cuerpo", ni "el mundo y el cuerpo dentro de vidente"; 160 no es ni una visibilidad primera que ve los 'quale' y las cosas, ni una visibilidad segunda que ve líneas de fuerza y dimensiones, ${ }^{161}$ sino que se apunta a "una misma corporeidad o visibilidad en gene$\mathrm{ral}^{\mathrm{H}} .162$

El cuerpo estesiológico es un "ser sonoro", 163 y aunque estoy "siempre del mismo lado del cuerpo", 164 y "oigo mi vibración desde dentro", 165 cuando "me siento bastante cerca del otro... casi asisto... al terrible nacimiento de la vociferación" ${ }^{166}$ En la estructura del mundo mudo del cuerpo "se da ya toda posibilidad de habla". 167

Sin embargo, el cuerpo estesiológico, por ser carne, "es pasivoactivo (visible-vidente), masa en sí y gesto"; 168 de hecho, nos muestra la reversibilidad y el cruce. En este sentido el ejemplo de

\begin{tabular}{|c|c|c|}
\hline $\begin{array}{l}149 \text { p. } 246 . \\
150 \text { p. } 312 . \\
151 \text { p. } 194 . \\
152 \text { p. } 313 . \\
153 \text { p. } 189 . \\
154 \text { p. } 179 . \\
155 \text { p. } 309 .\end{array}$ & $\begin{array}{l}{ }^{156} \text { p. } 200 . \\
{ }^{157} \text { p. } 280,284 . \\
{ }^{158} \text { p. } 194 . \\
{ }^{159} \text { p. } 192 . \\
{ }^{160} \text { p. } 182 . \\
{ }^{161} \text { p. } 195 . \\
{ }^{162} \text { Ibid. }\end{array}$ & $\begin{array}{l}163 \text { p. } 190 . \\
164 \text { p. } 326 . \\
165 \text { p. } 190 . \\
166 \text { Ibid. } \\
167 \text { p. } 203 . \\
168 \text { p. } 324 .\end{array}$ \\
\hline
\end{tabular}


la mano que toca a la otra ${ }^{169}$ que se repite una y otra vez, viene a mostrarnos los "dos segmentos de un solo recorrido circular", 170 la inserción recíproca de cuerpo sentido y cuerpo sintiente. ${ }^{171}$ Allí tenemos la intercorporeidad en que hay un círculo: tocantetocado, vidente-visible y recíprocamente; hay una inscripción: de lo tocante en lo tocado, del vidente en lo visible y recíprocamente; hay propagación: de intercambios a todos los cuerpos del mismo tipo y del mismo estilo. ${ }^{172}$

El cruce lo tenemos en que "lo tocante no es nunca exactamente lo tocante", 173 "la unión tocante-tocado se hace por el Pensamiento o Consciencia: el Pensamiento o Consciencia es Apertura de una corporeidad al... Mundo o Ser" ${ }^{174}$ Sin embargo, Merleau-Ponty nos ofrece la solución, para el caso del cuerpo, en que las manos "pertenecen a un solo espacio de consciencia", 175 en que es "un solo hombre el que toca... mediante sus dos" manos, ${ }^{176}$ y en que las manos son "manos de un mismo cuerpo". ${ }^{177}$ Mi cuerpo es un "cuerpo sinergético" y tiene una "unidad pre-reflexiva y pre-objetiva". 178

\section{Problemas de realización a escala de nuestra came}

Merleau-Ponty enraíza su filosofía en un aspecto poco conscientizado dentro de la filosofía occidental: la carne no había sido tomada como tema de reflexión filosófica a ese nivel. Precisamente por eso, hay algunos problemas que Merleau-Ponty no estuvo en posición de vislumbrar, aun cuando no podamos decir que se haya encaminado en dirección equivocada.

Quiero aquí solamente presentar algunos problemas en relación con el cuerpo estesiolósigo-carne nuestra (que llamaremos aquí cuerpo-carne), que Merleau-Ponty no parece haber llegado a conscientizar. Estos problemas ciertamente desde un punto de vista de "lo natural es ya perfecto", no se presentan. Sin embargo, la manera ordinaria de ser cuerpo-carne del hombre occidental y del hombre oriental nos llevan a ver la necesidad de explicitar la dualidad que choca con la no-dualidad a que MerleauPonty quiere conducirnos.

Todos damos por sobreentendido que el cuerpo humano tal co-

$\begin{array}{lll}{ }^{169} \text { p. } 24,182, & { }^{172} \text { p. } 188 . & \\ 185,209,307, \text { etc. } & 173 \text { p. 30\%. } & { }^{176} \text { p. } 186 . \\ 170 \text { p. } 182 . & 174 \text { p. } 308 . & { }^{177} \text { Ibid. } \\ 171 \text { Ibid. } & 175 \text { pp. } 185-186 . & { }^{178} \text { Ibid. }\end{array}$


mo se vive es ya perfecto y capaz aún de ser base de un des-velamiento del "Ser Salvaje" como lo piensa Merleau-Ponty. Pero allí hay un aspecto de inconsciencia en la filosofia occidental frente al cuerpo.

Al empezar la práctica de todo deporte, aun cuando estemos sanos, no por eso nuestro cuerpo está "en forma". Aun cuando pueda mover los dedos rápidamente en el teclado de una máquina, eso no me da una seguridad en los matices por presión digital en el teclado de un piano. Quizá la ciencia que busca la "objetividad $^{n}$ fue la primera en percatarse de la subjetividad ocasionada por la presencia de bulto del observador. Pero aún allí queda sin analizar una gran área de la corporalidad humana. Una filosofía que quiere poner también al cuerpo-carne como base de un des-velamiento del Ser bruto, con mayor razón tiene que conscientizar estos problemas. Por esto mismo tenemos que ver algunas de las características que ese cuerpo generalmente tiene y que no abandona en el vivir diario por no hacer del vivir diario su camino.

Tanto orientales como occidentales viven en la vida diaria:

a) Un cuerpo que se pone tieso en presencia de otro. En cuanto alguien nos lo haga notar nos daremos cuenta de ello; sin embargo, ordinariamente no estamos conscientes de esto. Lo podemos observar en muchos animales, especialmente en el gato y el perro a los que se les eriza el pelo, los caballos tienen contracciones musculares (especialmente de los tejidos subcutáneos), el gallo pone tieso el cuello, etc. Nuestro cuerpo también reacciona igual y se pone tieso en presencia de otro. Hay en ello ya un establecimiento de dualidad, un choque con el aura ajena, una carga bioeléctrica que ocasiona tensión muscular. En casos extremos produce agitación, dilatación o contracción vascular, atragantamiento salival, contracciones intestinales, etc. Un cuerpo ajeno causa nerviosismo. "Presentimos" un cuerpo ajeno por la carga bioeléctrica que induce en nosotros. Pero ese "presentir" difiere de la "auditividad dermal" de un cuerpo entrenado. Es una diferencia, se dice, de "estado" corporal, de "condición". Pero hay allí algo más profundo que eso y que sólo se conoce en la experiencia del "soltar el cuerpo y la mente" de la experiencia Zen, en el "morir al hombre viejo", o en el "morir con Cristo y resuscitar con Cristo" del cristianismo.

b) Un cuerpo que no está igualmente conscientizado en todas sus partes. Esto quiere decir que vivimos esencialmente nuestra vida en partes conscientes y partes inconscientes de nuestro cuerpo. Hay por esto también allí dualidad. Basta con sentarse ante 
un órgano para darse cuenta de ello; basta con entrenar un poco cualquier arte marcial para darse cuenta de que hay partes del cuerpo que en la vida diaria no ocupamos. Sin embargo, aquí no se habla de una apropiación intelectual de una carne, no es el problema de una "incorporación" en el sentido de dos elementos constitutivos: carne y consciencia, que tengan que unirse. Más bien, el hecho de que así se vea ordinariamente el problema nos apunta a un estado de dualidad en que generalmente al crecer se va habituando el cuerpo. Gran parte de la publicidad, mercadeo, etc. en representaciones visuales se dirigen a esta región no conscientizada de la humanidad. Pero aquí no se habla de una cierta destreza, sino de un desarrollo de las facultades ordinarias. Algunos deportes favorecen el desarrollo de unas, otros el de otras. Asimismo al escribir alfabetos y al escribir caracteres chinos el tipo de movimientos que conscientemente se tienen que hacer difiere. Ciertamente del grado en que el cuerpo está "despierto" en sus facultades ordinarias depende también su integración como cuerpo humano. Al hablar de ese "despertar", no sólo hacemos referencia a las partes móviles del cuerpo sino también a aquéllas que no se mueven hacia el exterior. Por otra parte, no hablamos de una preocupación mórbida por el cuerpo, o de un narcisismo a la $n$ potencia.

c) Un cuerpo que es alternativamente pasivo y activo. Si podemos interpretar de alguna manera el llamado "deseo corporal", el cuerpo que conoce el deseo carnal, aquí es donde lo podemos hacer. Aquí está también el hiatus, cruce, que Merleau-Ponty no logró sino apuntar en sus escritos. ${ }^{179}$ Con un cuerpo así las palabras aquéllas, "el espíritu está pronto pero la carne es débil" no logran entenderse sino en un sentido de una dualidad cuerpo-psique, o cuerpo-alma; que después metafísicamente trata de borrarse como quien tapa un hoyo ontológico. Un cuerpo que alternativamente es pasivo y activo está sumergido en su facticidad y en una temporalidad cronométrica. Un cuerpo que alternativamente es pasivo y activo, y aún aquél que sin dejar de serlo logra en un salto mortal activamente ser pasivo, no logran descifrar el "no me toques" de Jesucristo. ${ }^{180}$ Por estar envuelto en la dualidad de lo activo y lo pasivo es un cuerpo que en las artes marciales aún no es capaz de llevar sobre sí la carga emocional que en el piano se expresa a través de la sonoridad de teclado, dedos, cuerdas y pedales; en una palabra, aún está fraccionado, como lo veremos ahora.

${ }^{179}$ pp. 172 ss. $\quad \quad{ }^{180} \mathrm{Jn} 20: 17$. 
d) Un cuerpo que se vive esparcido multidireccionalmente. Quien ha tratado de hacer levantamiento de pesas viene a darse cuenta de que el levantamiento se efectúa no sólo por la potencia muscular, y que al principio hay una pérdida de energía que se escapa en direcciones distintas a la del levantamiento. Un luchador, boxeador, pitcher, golfista o karateka, conforme progresa en su entrenamiento va logrando disminuir esa pérdida por lateralidad. Es una situación corporal equivalente a una difusión multidireccional de vitalidad. Hay allí una dualidad de la que el movimiento en esos casos viene a hacernos conscientes. Un cuerpo así aún no es capaz de imprimir en la forma del carácter chino aquella coloración especial de la tinta que fluye en un pincel y que marca un punto decisivo en la evaluación de la caligrafia. Aún no es capaz de una buena caligrafia. Un cuerpo así aún no es capaz de comprender, sólo de repetir el levantamiento de un dedo. Aún no es capaz de comprender la respuesta de Jesús ante Pilato y en consecuencia interpretará el comentario sobre esto que San Pablo hace ${ }^{181}$ sólo como una ausencia de mancha o culpa, una irreprochabilidad de la conducta en sentido legal. Un cuerpo así aún no es capaz de seguir las instrucciones de Takuan; ${ }^{182}$ ni aún es capaz de respetar el principio aquél de "máxima eficiencia con mínimo de gasto de energía".

e) Un cuerpo empeñado en la lucha por la supervivencia. Ciertamente es un cuerpo natural que en ningún momento se separa de su habitat si no es por la actividad consciente; pero como ya vimos, es un cuerpo de dualidad que comparte la dualidad generación-muerte de una visión de la naturaleza. No sólo eso, es un cuerpo que se apropia la vida como un derecho y teme la muerte como su máximo enemigo. No es un cuerpo que pueda ver y vivir el horizonte vida-muerte-resurrección si no es en un refugio de vida; escoge un rincón en ese horizonte y se apega a él. De ahí que un cuerpo así, originalmente tienda a una existencia "generativa". Por ser cuerpo de dualidad lleva una vida en un nivel diferente de la consciencia: es un cuerpo salvaje empeñado en la lucha por la supervivencia. La esencia de su existir es supervivencia - esa es la constante que le anima. De allí que analizado a fondo el proyecto de Merleau-Ponty, en la dimensión cuerpocarne, queda fundado sobre una dualidad aún no conscientizada. Es un cuerpo-carne de combate, no de paz.

${ }^{181}$ I Tim. 6:13.

${ }^{182}$ Takuan Zenji, Fudo-chi shin-myo roku. 


\section{CONCLUSIÓN}

La resolución del problema cuerpo-carne como realización a escala del proyecto filosófico de Merleau-Ponty podría encontrar una solución como la que ahora brevemente expresaremos así:

La posibilidad misma de la experiencia del Ser-Bruto se marca por un CAMBIO en mi manera de SER CUERPO y en la VIDA DE MI CARNE.

Para resumir, en la visión de Merleau-Ponty "la filosofía no es un léxico, no se interesa en la 'significación de las palabras', no busca un sustituto verbal del mundo, no lo transforma en cosa dicha, no se instala en el orden de lo dicho o de lo escrito... Quiere llevar a la expresión a las cosas mismas desde el fondo de su (i.e. de las cosas) silencio". ${ }^{183}$

Si la interpretación aquí presentada es correcta, podría servirnos de base para una posible aproximación al pensamiento del "padre de la filosofia japonesa moderna", Nishida Kitaro. Esta aproximación habría de tomar en cuenta que:

a) quizá en la historia de la filosofía es con Merleau-Ponty con quien se plantea más agudamente el problema de la corporalidad;

b) en Merleau-Ponty el problema de la corporalidad está encuadrado en el problema más general de la experiencia y manifestación del Ser Bruto;

c) el problema de la corporalidad está referido a una dialéctica: en esa dialéctica el "entre" y el "cruce" juegan un papel semejante a las Reglas de Transformación para el caso de Nishida;

d) el rechazo de la separación sujeto-objeto;

e) el entrelazamiento existencial de los seres;

f) el sujeto verdadero concebido como un campo; y principalmente

g) el papel central de la corporalidad tanto en la filosofia de Merleau-Ponty como en la de Nishida Kitaro.

${ }^{183}$ Le Visible et...p. 18. 